$$
\begin{array}{rr} 
& \text { GA-A--20577 } \\
\text { JAN } 131992 & \text { DE92 } 005442
\end{array}
$$

\title{
DESIGN OF A COIL TO CORRECT MAGNETIC FIELD ERRORS \\ ON THE DIII-D TOKAMAK
}

\author{
by \\ J.T. SCOVILLE and R.J. LaHAYE
}

NOVEMBER 1991

\section{GENERAL ATOMICS}




\section{DISCLAIMER}

This report was prepared as an account of work sponsored by an agency of the United States Government. Neither the United States Government nor any agency thereof, nor any of their employees, makes any warranty, express or implied, or assumes any legal liability or responsibility for the accuracy, completeness, or usefulness of any information, apparatus, product, or process disclosed, or represents that its use would not infringe privately owned rights. Reference herein to any specific commercial product, process, or service by trade name, tridemark, manufacturer, or otherwise, does not necessarily constitute or imply its endorsement, recommendation, or favoring by the United States Government or any agency thereof. The views and opinions of authors expressed herein do not necessarily state or reflect those of the United States Government or any agency thereof. 
GA-A20577

\title{
DESIGN OF A COIL TO CORRECT MAGNETIC FIELD ERRORS ON THE DIII-D TOKAMAK
}

\author{
by \\ J.T. SCOVILLE and R.J. LaHAYE
}

This is a preprint of a paper to be presented at the 14th Symposium on Fusion Engineering, September 30-October 3, 1991, San Diego, California, and to be printed in the Proceedings.

\author{
Work supported by \\ Department of Energy \\ Contract DE-AC03-89ER51114
}
GENERAL ATOMICS PROJECT 3466
NOVEMEER 1991

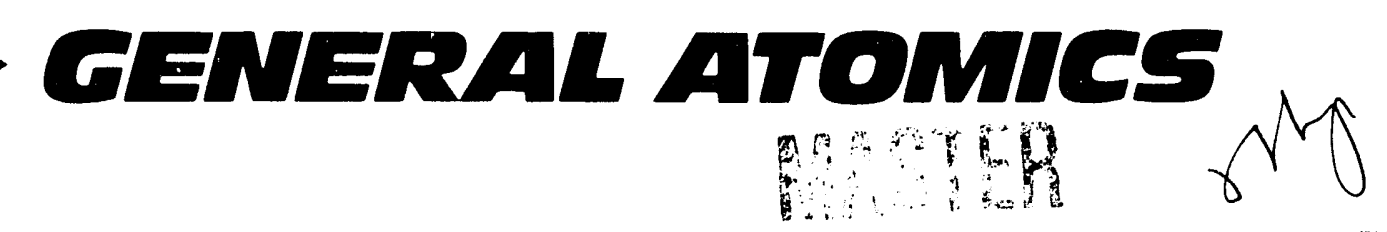




\title{
DESIGN CF A COIL TO CORRECT MAGNETIC FIELD ERRORS ON THE DIII-D TOKAMAK
}

\author{
I.T. Scoville and R.J. La Haye \\ General Atomics \\ P.0. Box 85608 \\ San Diego, California 92186-9784
}

Abstract: Small toroidally asymmetric errors on the ideally symmetric magnetic field of a tokamak are of concern for the design of next-generation devices and the performance of existing machines. Errors as small as one part in $10^{4}$ or less can be enough to cause detrimental effects on the stability and confinement of the plasma. Previously, we have used a simple perturbation coil mounted on top of the DIII-D tukamak (" $n=1$ coil") to enhance or partially reduce the error fields, resulting in significant effects on the stability of the plasma. Recently, careful measurements were made of the error fields created by the 18 field-shaping coils on DIII-D. Spectral analysis has yielded a better understanding of the source of the toroidal and polcidal field errors existing on the tokamak. With this new information, a much better error-field correction coil ("C-coil") has been designed which should be able to nearly eliminate the $m=2, n=1$ component of the error field without introducing other toroidal modes. By dividing the $\mathrm{C}$-coil into segments which can be connected in different configurations, we will be able to program the phase and amplitude of a variety of perturbation fields for experimentation. Alternatively, the coil can be configured and programmed to provide the maximum reduction of error fields for routine plasma operations, enabling a wicler stable operating parameter space for the tokamak. Details of the C-coil design and its harmonic spectrum are presented.

\section{Introduction}

As progress is made in tokamak fusion energy research, the importance of small errors or asymmetries in the magnetic field is becoming more evident. Small errors in the location of poloidal field coils with respect to the magnetic center of the plasma, for example, will produce magnetic islands within the plasma $[1,2]$. These resonant field errors can reduce the energy confinement, cause the growth of instabilities, lower the plasma rotation speed, and produce asymmetries in the heat flux to the vessel walls [3]. For future devices with much larger power flux densities, the consequences of unexpectedly high heat fluxes could be serious and there is theoretical evidence that, for some planned future devices, the sensitivity to error fields will increase [4]. By cltering the magnitude and/or phase of the error fields of a tokamak, useful information can be acquired about the sensitivity of the plasma to perturbations on the uniform, symmetric magnetic field.

A cross section of the DIII-D tokamak showing all the poloidal field shaping coils (F-coils) is presented in Fig. 1. Also shown, mounted above the center of the vessel on one side of the tokamak, is the " $n=1$ coil." This coil has been used to increase or decrease the error field of the tokamak and is routinely used during plasma operations to expand the stable operating parameter space of DIII-D [3]. The location of the coil and the toroidal and poloidal mode spectrum it produces, however, are not ideal.

Recently, the magnetic field components of each of the F-coils were measured with a set of orthogonal pickup coils placed at the center of the DIII-D vessel $[5-7]$. The pickup coils were aligned magnetically with the toroidal field and used to determine the vertical and radial error field components for the toroidal modes $n=1$ and $n=2$ for each $F$-coil. The results of these measurements have led to the design of a new error ficld correction coil (the "C-coil") which can much more closely match the error field spectrum of DIII-D without introducing potentially harmful sidebands. Besides improving on the $n=1$ coil's ability to reduce errors, the $\mathbf{C}$-coil design also provides control of the phase of the applied correction field.

The construction and installation of the C-coil on DIII-D is planned for early 1992, with routine operation to commence shortly afterward. We expect the improved error field compensation capability of the coil to lead to an expanded operating parameter space (in density, for example) and possible improvement in various plasma parameters such as stored energy, confinement time, and temperature. From previous experiments, a reduction of resonant magnetic field errors is expected to result in increased tearing mode stability and an improved ability to avoid locked modes [3]. The most important goal of the planned C-coil experiments is to gather information on the effect of error fields on plasma parameters which will aid in the design of next generation devices.

\section{Poloidal Field Coil Errors}

Non-rotating magnetic perturbations called "locked modes" can be found over a wide range of parameter space on the iokamak. Measurements made by saddle loops mounted on tise DIII-D midplane indicate that these stationary magnetic islands have a preferential toroidal location, caused by the existence of toroidal asymmetries in the magnetic field. On DIII-D, we have determined that these asymmetries are caused by slight errors in the location of the poloidal field coils with respect to the toroidal field.

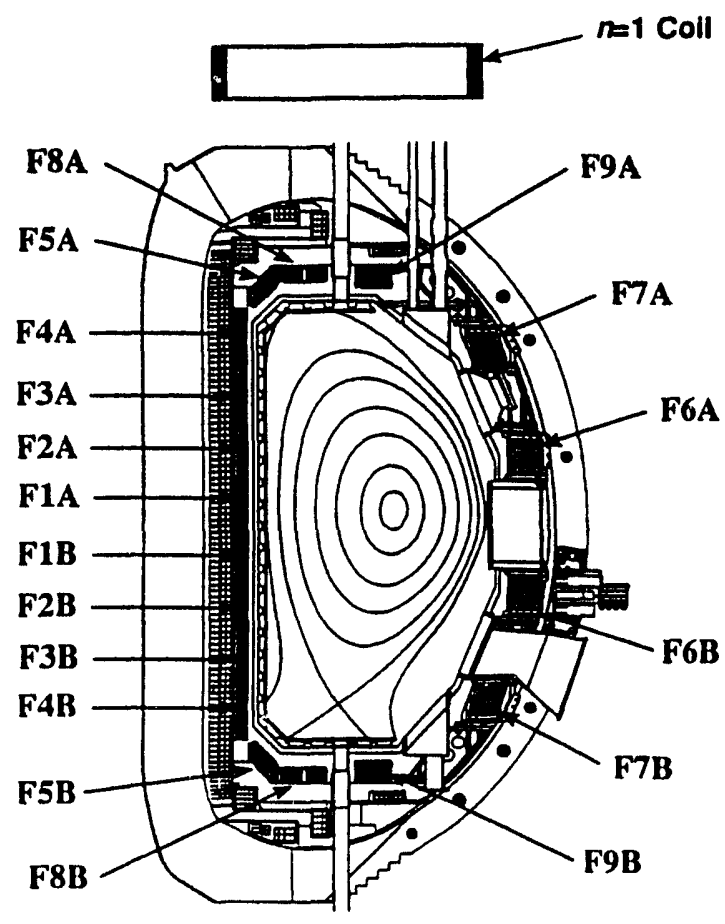

Fig. 1. Cross section of the DIII-D tokamak showing flux surfaces, F-coils, and the $n=1$ error field coil. 
In an attempt to understand the sensitivity of the plasma to these very small field errors, the magnetic field of each of the F-coils was carefully measured. By connecting pickup coils located at the center of the vessel in pairs either $180^{\circ}$ or $90^{\circ}$ apart, the sine and cosine components for both radial and vertical field were measured. Using Fourier analysis, the error fields produced by each of the F-coils for the first two toroidal modes ( $n=1$ and $n=2$ ) were deduced [5]. For $n=1$, the error fields determine the shift and tilt of the coil; for $n=2$, we can calculate the stretch and bow, or the ellipticity of the coil. We find that the most irregular poloidal field coil is one of the large outer vertical field coils. The error produced by this coil corresponds to a shift of $1.9 \pm 0.2 \mathrm{~cm}$ (compared to a diameter of $482 \mathrm{~cm}$ ) and an ellipticity of 1.0027 .

During a plasma discharge, the $18 \mathrm{~F}$-coils are each independently controlled, carrying a current determined by the desired equilibrium. A typical discharge shape for DIII-D is the single-null divertor (SND), as depicted by the flux surfaces shown in Fig. 1. The $n=1$ radial error field components produced at the vessel center by each F-coil are shown in Fig. 2 for a typical 0.95 MA, SND discharge (\#65489). Results of Fourier analysis of the F-coil crrors for this SND discharge are plotted in Fig. 3, showing the poloidai mode spectrum for the two toroidal modes $n=1$ and $n=2$. Notice that the amplitudes of the $m=1, n=1$ and $m=2, n=1$ modes are greater than $2 \mathrm{G}$. In some cases, especially in discharges with a very low safety factor $(q)$ and/or low density, these mode amplitudes are large enough to trigger plasma instabilities. In many instances, for example, considerable MHD activity with $m=2$ and $n=1$ is observed prior to plasma disruption [8].

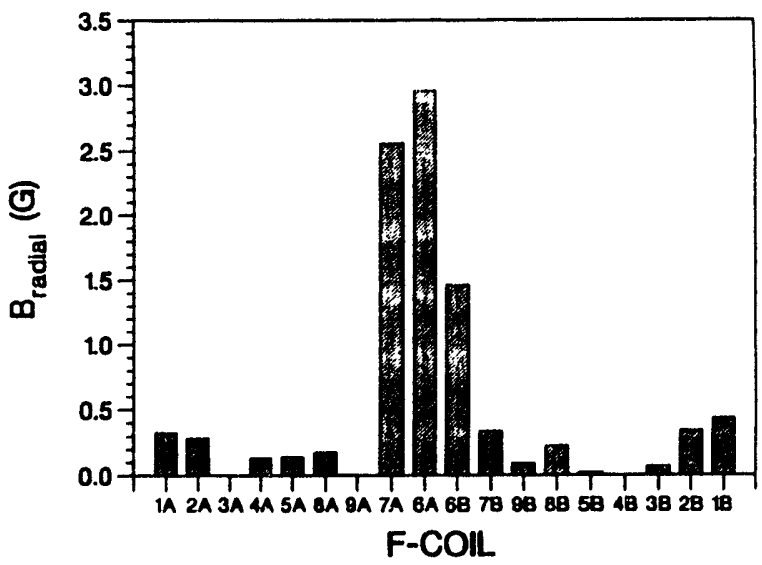

Fig. 2. Radial $n=1$ errer fields measured at vessel center for different F-coils. SND discharge \#65489.

Error field analysis is done by a code which requires the coordinates of the coils in question. The coil currents are broken up into many short, straight segments and the Biot-Savart law is applied; the resulting field is represented as a double harmonic series of left- and right-handed helical mode components. For a given discharge, the $\mathrm{F}$-coil currents are known and combined in a code which does the Fourier analysis in separable toroidal coordinates on a toroidal surface the size of DIII-D [3]. The result is a table of coefficients of the sine and cosine components for mode numbers $n=1-9$ and $m=0-11$, from which the amplitude and phase information is obtained.

\section{The " $n=1$ Coil"}

The significance of the $n=1$ error field was investigated during the early $n=1$ coil experiments on DIII-D [3]. The modest reduction in the $n=1$ field enabled by the coil permitted stable operation in a low density regime which was previously unattainable because of the onset of locked modes. The $n=1$

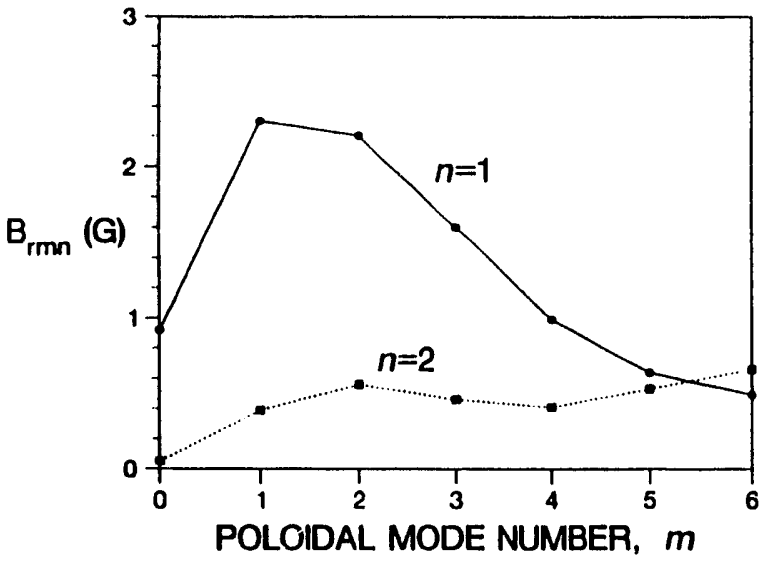

Fig. 3. Poloidal mode spectrum for $n=1$ and $n=2$ showing total radial error field components of all F-coils for shot \#65489.

coil, however, is not the ideal correction coil; although the spectrum it generates has a large fraction of $n=1$, it contains a significant $n=2$ component and a full compliment of poloidal modes (see Table 1 ). As a result, the optimum $n=1$ coil current to minimize one mode can increase another. This is shown in Fig. 4, where, for discharge \#65489, the $m=2, n=1$ mode has been considerably reduced by the $n=1$ coil while some of the other poloidal components are increased.

\section{A New Correction Coil}

In order to reduce the detrimental effects of the error fields of the tokamak, a magnetic field must be generated which cancels the error field for the modes of concern. For DIII-D and other tokamaks, the modes of primary concern usually have $n=1$ and $m=2$ or $m=3$. There are several ways to produce the necessary field, but t'ie design must be compatible with existing equipment already installed on the tokamak. Some of the factors which determined the design of the C-coil include space constraints near the machine, a practical method for attaching and holding the coil, and a desire for the field to be radial at the midplane, since it is the field normal to the flux surface which produces magnetic islands. The resulting coil design consists of six separate sections, each $60^{\circ}$ wide toroidally, connected in series and encircling the tokamak toroidally at midplane (see Fig. 5). Each of the six sections includes four turns of $250 \mathrm{MCM}$ cable wound so that the upper and lower legs of the coil are $\pm 0.8 \mathrm{~m}$ from the vessel midplane.

The C-coil must be mounted as close to the plasma as possible in order to produce the desired magnetic field with a minimum of amp-turns. Fewer amp-turns means a smaller, more manageable coil and lower power supply requirements. For DIII-D, the best location available is just outside the toroidal field coil. The upper and lower legs of the coil will be secured every $15^{\circ}$ by mounting arms attached to the F7A and F7B F-coil casings. These rigid arms provide support, approximately every $80 \mathrm{~cm}$ circumferentially, against the $\mathrm{J} \times \overline{\mathrm{B}}$ forces resulting from

Table 1

Radial Field Spectrum of the $n=1$ Coil Values are in Gauss for 140 kA-Turns in the Coil

\begin{tabular}{lcccccc}
\hline & $m=0$ & $m=1$ & $m=2$ & $m=3$ & $m=4$ & $m=5$ \\
\hline$n=1$ & 1.01 & 6.44 & 2.38 & 0.84 & 0.34 & 0.14 \\
$n=2$ & 0.14 & 2.35 & 1.20 & 0.50 & 0.20 & 0.08 \\
$n=3$ & 0.06 & 0.53 & 0.36 & 0.20 & 0.08 & 0.03 \\
\hline
\end{tabular}




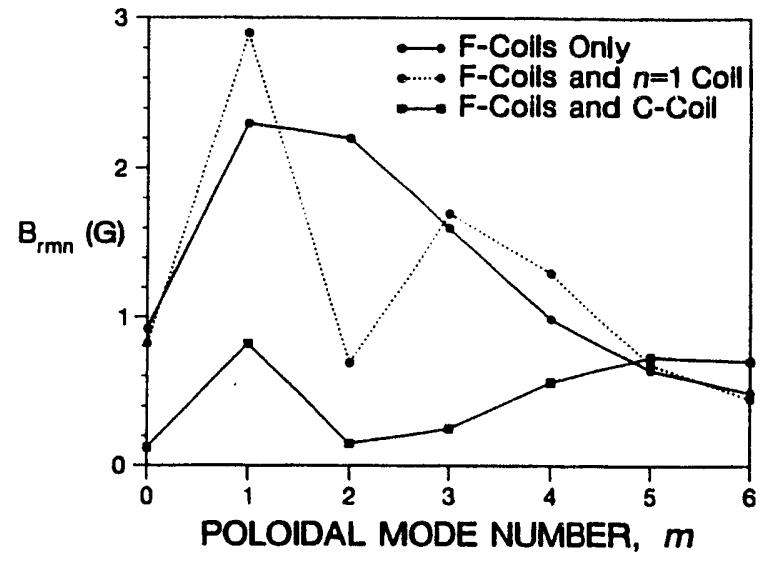

Fig. 4. Poloidal mode spectrum for $n=1$ showing radial error fields of $F$-coils alone and combined with the $n=1$ coil or the C-coil for shot \#65489.

the interaction of the tokamak's poloidal field with the primarily toroidal C-coil current. The routing of the coil windings will, in some cases, not be ideal; flexibility is required in order to avoid the many systems attached to the tokamak. A variation of a few centimeters in the horizontal or vertical location of the windings will produce about $2 \%$ error in the applied field. Any larger variations in coil location will be incorporated into the code which calculates the resulting error fields.

For plasma currents up to $3 \mathrm{MA}$, the maximum $\mathrm{C}$-coil current required to minimize the tokamak's intrinsic $n=1$ error field is about $5 \mathrm{kA}$. With four turns of $250 \mathrm{MCM}$ cable in each of the six C-coil sections, the total resistance and inductance are approximately $25 \mathrm{~m} \Omega$ and $200 \mu \mathrm{h}$, respectively. Requiring a coil response time on the order of the energy confinement time $(\approx 100 \mathrm{~ms})$ provides an estimate of the required coil voltage approximately $130 \mathrm{~V}$. Any of the existing $\mathrm{F}$-coil dc power supplies can provide this power, or a dedicated $\mathrm{C}$-coil power supply $(200 \mathrm{~V}, 5 \mathrm{kA})$ could be acquired.

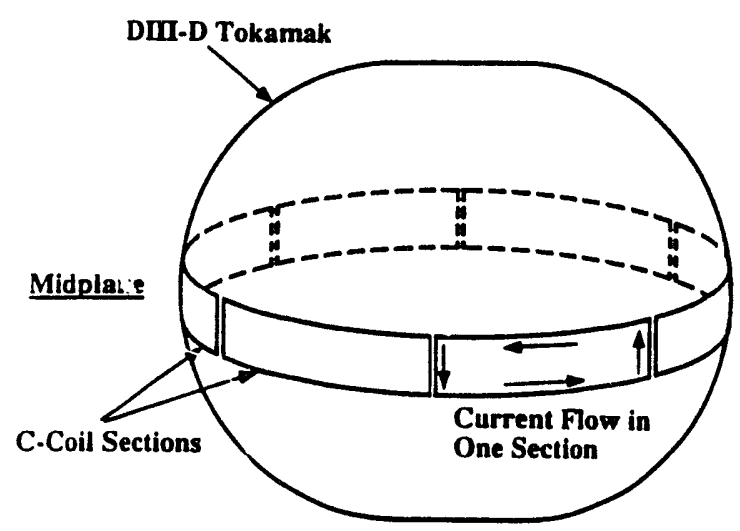

Fig. 5. Six sections of C-coil shown encireling the DIII-D tokamak at midplane.

\section{Phase Conirol}

Although it is not possible to cancel all error field modes simultaneously, the C-coil can reduce several of the low $m$ modes and virtually eliminate the $m=2$ mode if energized with the appropriate current and phase for a given discharge. Dividing the coil into six segments provides the flexibility necessary for phase control of the induced field. The "standard" C-coil configuration induces an $n=1$ field by energizing two consecutive sections with positive polarity and the opposite two with negative polarity. The phase of the applied field for this scheme would be limited to one of six values, $60^{\circ}$ apart. Without using six separate power supplies, considerable added flexibility can be obtained by taking advantage of the multi-turn nature of each $\mathrm{C}$-coil section winding. By adding three patch panels to the system, every $120^{\circ}$ between pairs of neighboring sections, the four turns of each section can be isolated to allow for connection of one, two, three, or all four (see Fig. 6). In this way, the current in each section can be wired to one of nine values. If the current fed to the $\mathrm{C}$-coil is $2 \mathrm{kA}$, for example, each section could carry $0,2,4,6,8,-2,-4,-6$, or -8 kA-turns. A considerable number of configurations are possible, allowing for the production of different toroidal modes and providing phase control.

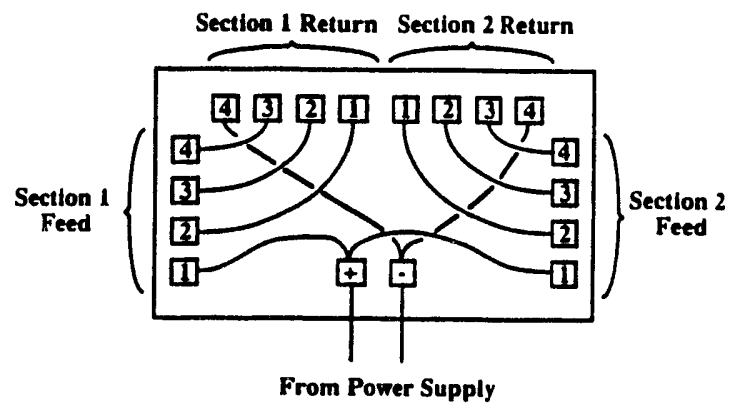

Fig. 6. One of three patch panels between neighboring C-coil sections, used to wire each section for one of nine values of current. The connections shown have each section carrying full positive current.

An example of how the applied phase can be controlled is shown in Fig. 7. The number of turns in three consecutive sections of the $\mathrm{C}$-coil is varied so that the $n=1$ amplitude remains relatively constant and little $n=3$ component is produced. (The current in the other three sections is equal and opposite, to produce $n=1$.) The phase is "steered" by connecting different numbers of turns from section to section, allowing for control of the phase to within approximately $4^{\circ}$. By choosing the appropriate three sections, the maximum of the applied radial field can be located at the desired azimuth. To optimize the effectiveness of the coil, the planned discharge shape can be studied, the resulting $\mathrm{F}$-coil currents deduced, and the $\mathrm{C}$-coil configured before the experiment to provide the best possible

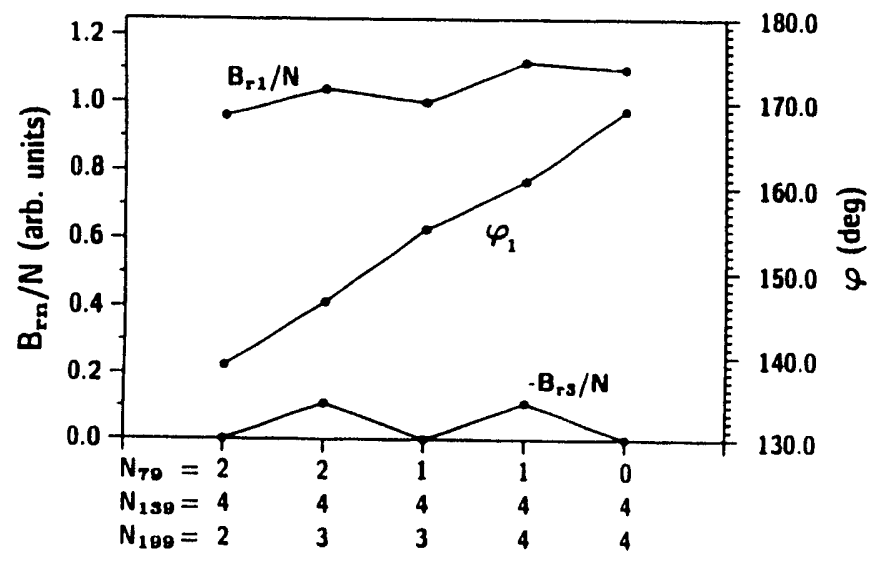

Fig. 7. By connecting different numbers of turns in neighboring $\mathrm{C}$-coil sections, the phase can be controlled. The $n=$ 1 amplitude is fairly constant and little $n=3$ is gen. erated. The centers of the sections are located at $79^{\circ}$. $139^{\circ}$, and $199^{\circ}$. 
error field cancellation. This will provide a useful tool for the experimentalist to help avoid locked modes, reduce the plasma's sensitivity to various MHD instabilities, and increase the stable operating parameter space of the tokamak.

\section{Improved Error Field Correction}

The improvement of the $\mathrm{C}$-coil over the $n=1$ coil in cancelling some of the components of the intrinsic error field of the tokamak is evident in Fig. 4. For the SND discharge analyzed, all the low $m, n=1$ modes are considerably reduced by the C-coil, especially the troublesome $m=2, n=1$ mode. Since much of the detrimental MHD activity seen before plasma disruptions is associated with $n=1$ and low $m$, we expect that routine operation with the $\mathrm{C}$-coil will allow for an expanded operating parameter space of the tokamak. Figure 8 shows the dependence of the radial magnetic field on C-coil current for three modes in the typical SND discharge. For this $0.95 \mathrm{MA}$ plasma current case, approximately $1100 \mathrm{~A}$ of C-coil current will significantly reduce the $m=1$ and $m=3$ modes and almost completely eliminate the $m=2$ mode.

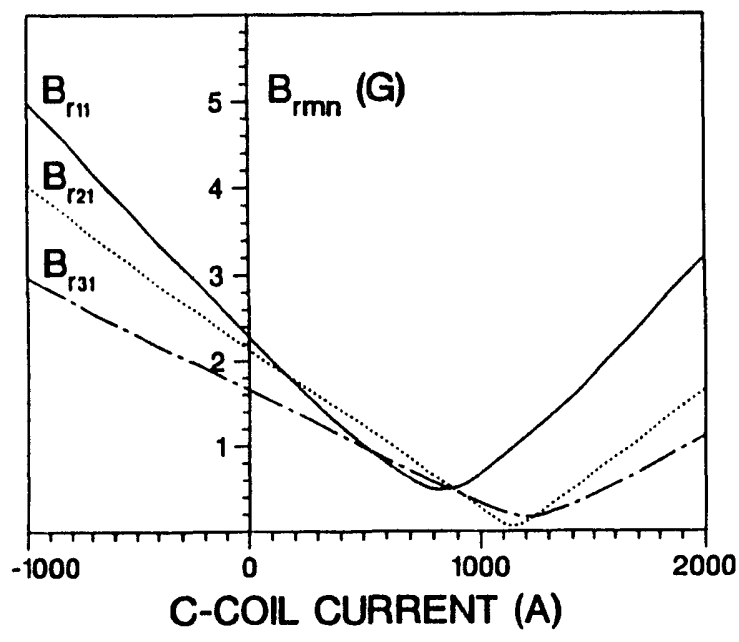

Fig. 8. The helical radial field for $n=1, m=1,2,3$ is minimized with abour $1100 \mathrm{~A}$ in the $\mathrm{C}$-coil. SND discharge \#65489.
If enough flexibility is built in to the lead design and power supply system, the $\mathrm{C}$-coil will provide the opportunity to carry out some very interesting experiments, including MHD feedback, locked mode control, and investigation of phase steering effects. The results of these and other experiments will be useful to those currently working on the design of next generation devices, since those machines may have a greater sensitivity to small field errors.

\section{Acknowledgment}

This work was sponsored by the U.S. Department of Energy under Contract No. DE-AC03-89ER51114, and sometimes other contracts or subcontracts through some of the collaborations.

\section{References}

[1] Colchin, R.J., J.H. Harris, D.K. Lee, and J.A. Rome, "Magnetic Field Alignment Studies for the URAGON-3 Torsatron," Rev. Sci. Instrum. 57 No. 7 (1986) 1233-1241.

[2] Brown, D.I., and D.M. Meade, "The PDX Island Study," Proc. Seventh Symposium on Eng. Problems in Fusion Research, Knoxville, Tennessee (1987) 1347-51.

[3] Scoville, J.T., R.J. La Haye, et al., "A Discussion of Locked Modes in DIII-D and a Method for Prevention of the Low Density Mode," Nuclear Fusion 31 No. 5 (1991) 875-890.

[4] Fitzpatrick, R., and T.C. Hender, "The Interaction of Resonant Magnetic Perturbations with Rotating Plasmas," Phys. Fluids B 3 No. 3, (1991) 644-673.

[5] La Haye, R.J., and J.T. Scoville, "A Method to Measure Poloidal Field Coil Irregularities in Toroidal Plasma Devices," General Atomics Report GA-A20356 (1991), submitted to Rev. Sci. Instrum.

[6] Jackson, G.L., J.S. deGrassie, et al., "A Method to Measure Magnetic Field Perturbations in Plasma Devices," Rev. Sci. Instrum. 60 No. 9 (1989) 2883-2887.

[7] Crossland, R.T., R.J. Hayward, et al., "COMPASS TF Coil Dynamic Vertical Pre-load Device and PF Coil Alignment Using a Fixed Coil Array," Symp. on Fusion Technology (SOFT) Conference, London (1990).

[8] Osborne, T.H., A.G. Kellman, L. Lao, et al., Bull. Am. Phys. Soc. 32 (1987) 1896. 

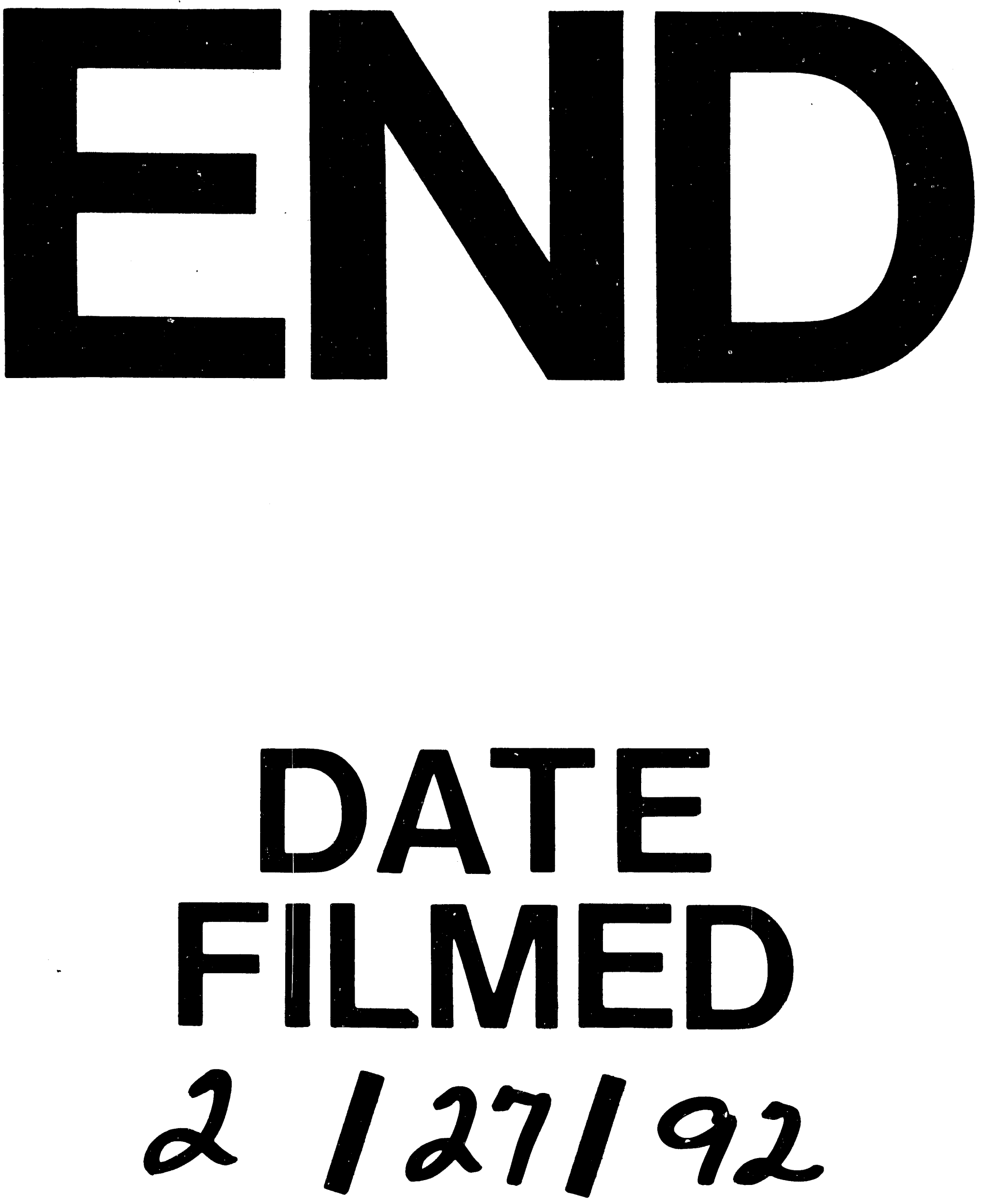
